\title{
A Component Model for Field Devices ${ }^{\star}$
}

\section{Oscar Nierstrasz ${ }^{1}$, Gabriela Arévalo ${ }^{1}$, Stéphane Ducasse ${ }^{1}$,} Roel Wuyts ${ }^{1}$, Andrew P. Black ${ }^{2, \star \star}$, Peter O. Müller ${ }^{3}$, Christian Zeidler ${ }^{3}$, Thomas Genssler ${ }^{4}$, and Reinier van den Born ${ }^{5}$

1 Software Composition Group, Institut für Informatik und Angewandte Mathematik, University of Bern, Switzerland, \{oscar, ducasse,wuyts\}@iam.unibe.ch,www.iam.unibe.ch/ scg/

2 Department of Computer Science \& Engineering, Oregon Health \& Science University, black@cse.ogi.edu,www.cse.ogi.edu/ ${ }^{\sim}$ black/

3 ABB Research Center, Germany, \{peter.o.mueller,christian.zeidler\}@de.abb.com, www.abb.com

4 FZI Research Center for Information Technologies, Germany, genssler@fzi.de,www.fzi.de

5 OTI, The Netherlands,

Reinier_van_den_Born@oti.com,www.oti.com

\begin{abstract}
Component-based software development is becoming mainstream for conventional applications. However, components can be difficult to deploy in embedded systems because of non-functional requirements. PECOS is a collaborative project between industrial and research partners that seeks to enable componentbased technology for a class of embedded systems known as "field devices". In this paper we introduce a component model for field devices that captures a range of non-functional properties and constraints.
\end{abstract}

\section{Introduction}

Software for embedded systems is typically monolithic and platform-dependent. These systems are hard to maintain, upgrade and customize, and they are almost impossible to port to other platforms. Component-based software engineering would bring a number of advantages to the embedded systems world such as faster development times, the reuse of existing components, and the ability for domain experts to interactively compose and adapt sophisticated embedded systems software. In order to apply CBSD to embedded systems, however, the following questions remain to be answered:

- Component models: What kind of component models are needed to support CBSD for embedded systems software?

- Non-functional requirements: How can we reason about non-functional constraints of systems based on properties of their constituent components?

- Tools: What tools are need to specify, compose, validate and compile embedded systems applications built from components?

The PECOS project ${ }^{* * *}$ aims to enable component-based software development for embedded systems. In order to achieve concrete results within a limited frame, PECOS is driven by a case study in the domain of field devices, which are field deployable con-

${ }^{\star}$ An extended version of this paper is available from: www.iam.unibe.ch/ $/ \mathrm{scg} /$.

${ }^{\star \star}$ Visiting SCG. 
trol devices briefly described in section 2. Section 3 presents a running example, and section 4 introduces the PECOS field device component model, focusing on the structural aspects. In section 5 we present the execution semantics of the component model, by translation to Petri nets. By extending this interpretation to time Petri nets [12], we intend to reason about real-time constraints and automatically generate real-time schedules. Finally, in section 6, we summarize the current state of the project, which is still in progress.

\section{P ECOS}

ABB's Instruments business unit develops a large number of different field devices, such as temperature, pressure, and flow sensors, actuators, and positioners. As field devices turn into commodities, the software increasingly determines the competitiveness of the devices. As the market demands new functionality in shorter time cycles, software begins to dominate the development and maintenance costs of field devices.

\subsection{Field devices}

A field device is a reactive, embedded system. Field devices make use of sensors to continuously gather data, such as temperature, pressure or rate of flow. They analyse this data, and react by controlling actuators, valves or motors. To minimize cost, field devices are implemented using the cheapest available hardware that is up to the task. A typical field device may contain a 16-bit microprocessor with only $256 \mathrm{kB}$ of ROM and $40 \mathrm{kB}$ of RAM.

The software for a typical field device, such as the TZID pneumatic positioner shown in figure 1 , is monolithic, and is separately developed for each kind of field device.

\subsection{P ECOS goals}

The goal of PECOS is to enable CBSD for embedded systems by providing an envi-

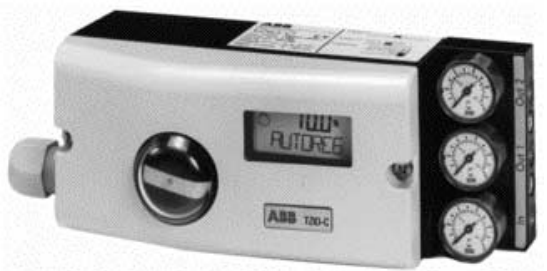

Figure 1 Pneumatic positioner TZID ronment that supports the specification, composition, configuration checking, and deployment of embedded systems built from software components.

By focusing on the field device case study, PECOS intends to deliver a demonstrator that validates CBSD for embedded systems. Specifically, PECOS intends to deliver both a component model suitable for characterizing software components for field devices, and a composition environment for expressing, validating and compiling compositions of components conforming to the model.

***Funded by the European Commission as project IST-1999-20398 and by the Swiss government as BBW 00.0170. The partners are Asea Brown Boveri AG (ABB, Germany), Forschungzentrum Informatik an der Universität Karlsruhe (FZI, Germany), Object Technology International AG (OTI Netherlands), Institut für Informatik und Angewandte Mathematik, University of Bern (UNIBE, Switzerland). 


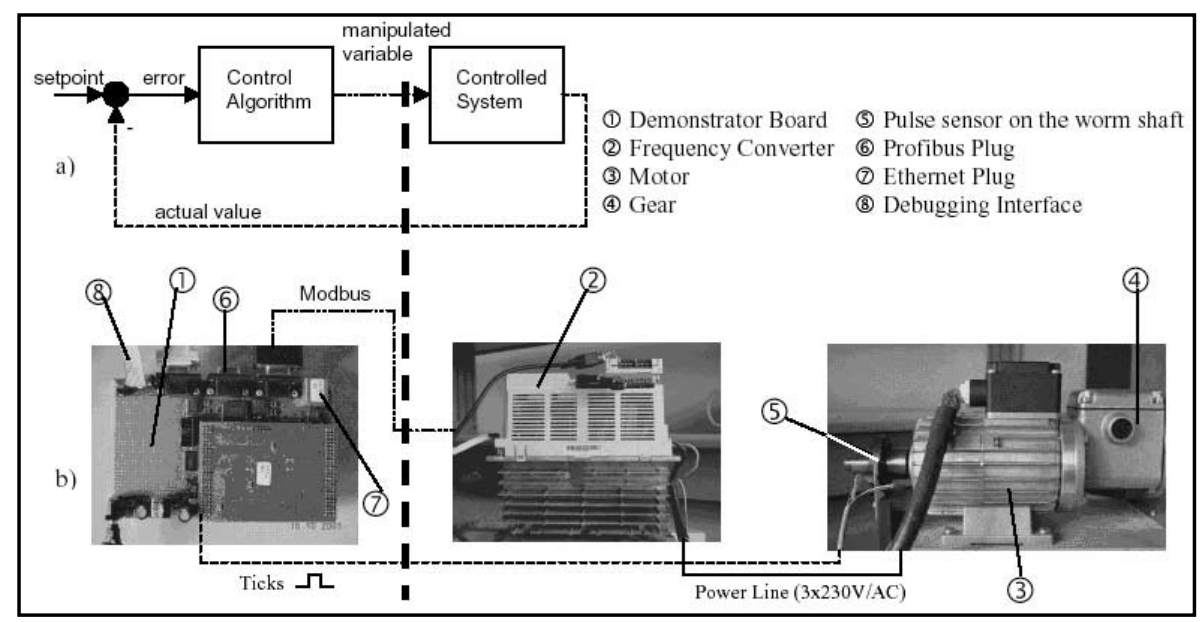

Figure 2 Pecos demonstrator field device

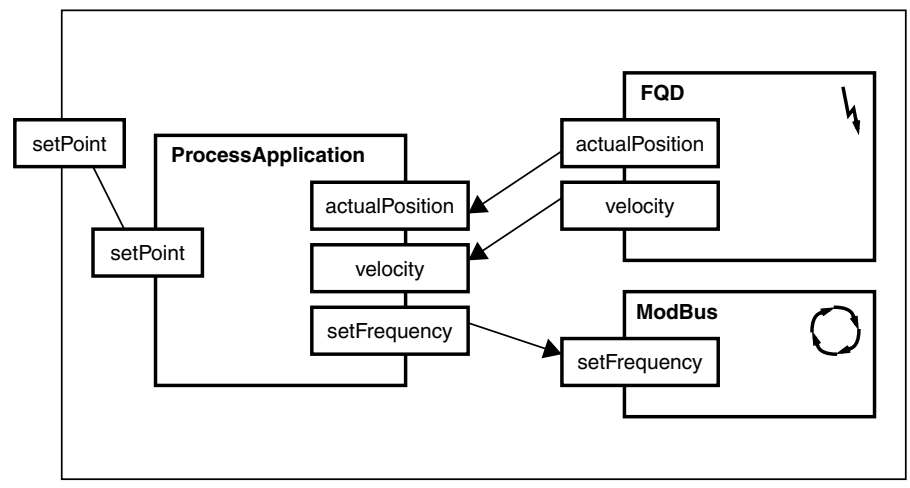

Figure 3 FQD Control loop example

\section{P ECOS Case Study}

In order to validate CBSD for embedded systems, the PECOS project is developing the hardware and software for a demonstration field device. The task of the PECOS field device is to control a three-phase motor connected to a valve (see figure 2). The motor is driven by a frequency converter which can be controlled over Modbus from the field device. The motor can be coupled to a valve either directly via a worm shaft or using additional gearing (4). A pulse sensor on the shaft (5) detects its speed and the direction of rotation. The PECOS board (1) is equipped with a web-based control panel (7) with some basic elements for local operation and display. The demonstrator can be integrated in a control system via the fieldbus communication protocol Profibus PA (6). The device is compliant to the profibus specification for Actuators [4][5].

\subsection{Running Example}

We will use the following example to illustrate the PECOS component model and composition language. 
Part of the PECOS case study is concerned with setting a valve at a specific position between open and closed. Figure 3 illustrates three connected PECOS components that collaborate to set the valve position; the desired position is determined by other components not shown here. In order to set and keep the valve at a certain position, a control loop is used to continuously monitor and adjust the valve.

- The ModBus component is responsible for interfacing to a piece of hardware called the frequency converter, which determines the speed of the motor. The frequency to which the motor should be set is obtained from the ProcessApplication component. ModBus outputs this value over a serial line to the frequency converter using the ModBus protocol (hence its name). The ModBus component runs in its own thread, because it blocks waiting for a (slow) response from the frequency converter.

- The FQD (Fast Quadrature Decoder [8]) component is responsible for capturing events from the motor. This component abstracts from a micro-controller module that does FQD in hardware. It provides the ProcessApplication with both the velocity and the position of the valve.

- The component ProcessApplication obtains the desired position of the valve (setPoint) and reads the current state of the valve from the FQD component. This information is then used to compute a frequency for the motor. Once the motor has opened the valve sufficiently, ascertained by the next reading from the FQD, the motor must be slowed or stopped. This repeated adjustment and monitoring constituted the control loop.

This example illustrates several key points concerning the field device domain.

- Cyclic behaviour: each component is responsible for a single task, which is repeatedly executed.

- Information flow through ports: components communicate by means of shared data. The interface of a component consists of a set of shared data ports.

- Threading: some components are passive, while others have their own thread of control.

- Separate scheduler: control flow is separately specified by a scheduler for the composite component.

\section{A Component Model for Field Devices}

The component model presented here has been especially tailored to the domain of field devices. Although it may have broader implications for other classes of embedded systems, we do not make that claim here. 
The PECOS field device component model has been defined to reflect an architectural style for field devices [9]. As such, we define a vocabulary of components, ports and connectors and the rules governing their composition. As in related approaches, components may only be connected if their provided and required ports are compatible [11].

Components. A component is a computational element with a name, a number of property bundles and ports, and a behaviour. The ports of a component represent data that may be shared

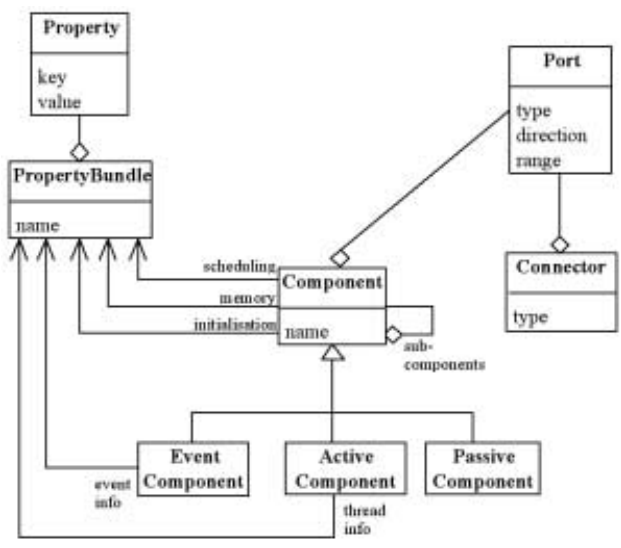

Figure 4 The PECOS Component Model with other components. The behaviour of a component consists of a procedure that reads and writes data available at its ports, and may produce effects in the physical world.

A leaf component is a "black-box" not further defined by model, but instead directly implemented in the host programming language. It has an interface consisting of a set of ports, and properties specified by its property bundles.

A composite component contains a number of connected subcomponents, the ports of which form the internal ports of the composite. A composite component also has external ports, which are the only ones that are externally visible. The external ports are connected to appropriate internal ports. The subcomponents are not visible outside the composite that contains them.

The field device domain requires three kinds of components.

- Passive Components do not have their own thread of control. A passive component is explicitly scheduled by the active component that is its nearest ancestor in the component hierarchy (its active ancestor). Passive components are typically used to encapsulate a piece of behaviour that executes synchronously and completes in a short time-cycle.

- Active Components do have their own thread of control; they are used to model ongoing or longer-lived activities that do not complete in a short time-cycle.

- Event Components are those whose behaviour is triggered by an event. They are used to model pieces of hardware that frequently emit events, such as motors that give their rotation speed, or timers that emit a timing event when a certain deadline has passed. Whenever the event fires, the behaviour is executed immediately.

Ports. A port is a shared variable that allows a component to communicate with other components; connected ports represent the same shared variable. A port specifies:

- a name, which has to be unique within the component;

- a type, characterizing the data that it holds; 
- a range of values (defined by a minimum and maximum value) that can be passed on this port; and

- a direction ("in", "out" or "inout") indicating whether the component reads, writes, or reads and writes the data. An inout port behaves exactly like a pair unidirectional ports, one in, and the other out.

Ports of peer components can only be connected if they have the same type and their direction is complementary, i.e., an in port can only be connected to an out port. Internal ports of a composite component can only be connected to the external ports if they have the same type and their direction is compatible, e.g., an internal in port can be connected to an external in port. Internal ports may be left unconnected, so it is allowed to connect an internal inout port to an external out port.

Connectors. A connector specifies a data-sharing relationship between ports. It has a name, a type, and a list of ports it connects. (Here we consider only binary connectors.)

Properties. A property is a tagged value. The tag is an identifier, and the value is typed. Properties characterise components.

Property bundles. A property bundle is a named group of properties. Property bundles are used to characterize aspects of components, such as timing or memory usage.

\subsection{Example revisited}

Returning to the example of figure 3, we see that FQD is an event component, ProcessApplication is a passive component and ModBus is an active component. The composition will be modelled as a composite component.

FQD has "out" ports actualPosition and velocity, connected to "in" ports of the same name belonging to ProcessApplication. The in port setPoint belonging to ProcessApplication is shared with the composite component that encapsulates this composition. It is not yet connected to a compatible "out" port. Finally, the "out" port setFrequency is connected to the "in" port of the same name belonging to ModBus.

\section{Synchronization and Timing}

Two issues must be addressed to complete the model: first, how read/write and write/ write conflicts are avoided on the (shared) external ports, and second, how components are scheduled to meet deadlines.

We will do this using a Petri net interpretation of valid compositions. Using plain Petri nets we can model concurrent activities of component compositions, scheduling of components, and synchronization of shared ports. This part of the model is reasonably well-understood. Using time Petri nets we hope to reason about timing constraints, and generate real-time schedules; this topic is still under investigation. 


\subsection{Synchronization}

Our Petri net procedures of the field device component model makes use of three different kinds of places and tokens. (i) Data places model ports; each data place has a single token representing the shared data available at that port. (ii) Control places are used to schedule components. Each active component has its own independent control subnet to model its schedule; there is exactly one token in each control subnet. (iii) Event places model the generation of an event.

A component is modelled as a Petri net fragment with a single control place that can be used to start it, and a single end place to signal that it has terminated (figure 5). When components are composed, a schedule must be generated that somehow moves the token from the end place of a component to the start place of the next one to be scheduled.

The behaviour of the component is a subnet that has read and write access to its data ports. The nature of these subnets depends on

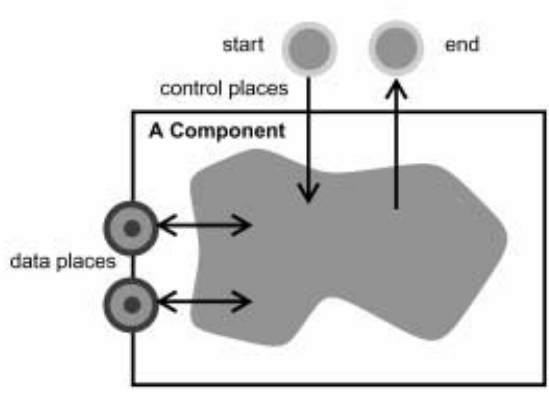

Figure 5 Components as nets the kind of component.

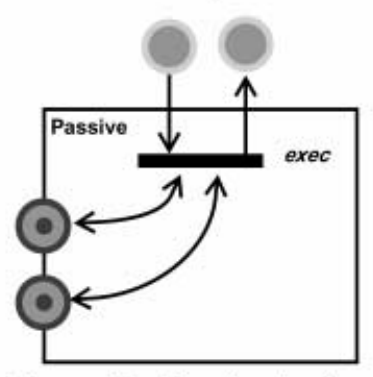

Figure 6 Passive leaf components

Passive leaf components are particularly simple to model. Their behaviour consists of a single exec transition that reads or write the data places (figure 6).

Because two passive components that share a port must be serialized, synchronization problems can arise only when active components are connected to other components. Active components compete for their external data ports with their surrounding environment. To address this problem, we split the external ports of active components into two parts: an outer port, to which the outside world has free access, and an inner port, to which the active component has access.

These two ports are synchronized by copying the data from one to the other (depending on the direction of the port) in a special synchronization method (or "sync method"). This method may be generated or specially tailored. We model this by a sync transition that reads and writes the inner and outer ports and is triggered by the start control place.

It is important to realize that the inner ports are actually the shared resources, since they are the only ones exposed to concurrent accesses (i.e., from the sync transition and from the in-

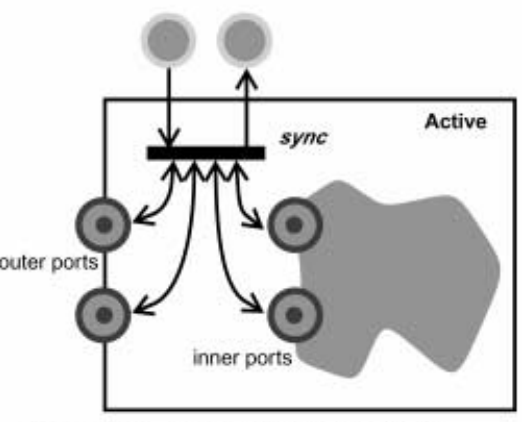

Figure 7 Synchronization of inner and outer ports ternal behaviour of the active components). 
The outer ports are never exposed to concurrent accesses, because they are only accessible from the transitions of the outer control net, which contains only a single token.

The behaviour of an active leaf component is modelled as a separate control subnet consisting of a critical section, which may access the inner ports, and a non-critical section. The control subnet of an active component is a loop containing a single control token.

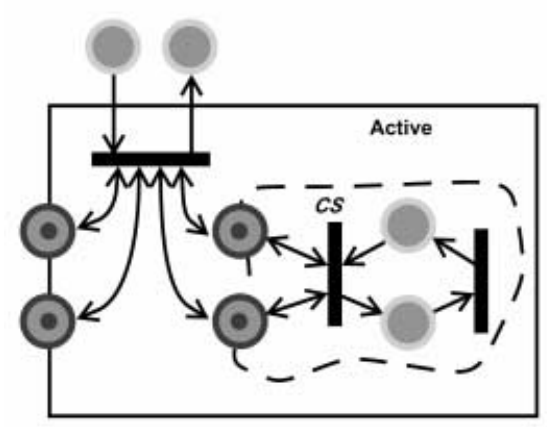

Figure 8 Active schedule with critical section

An event component is similar to an active component, except that its control subnet does not cycle, but is triggered by an external event.

To model composite components, we simply coalesce all the connected data places, and we connect the start and end control places of the subcomponents according to the required schedule.

Data ports may be connected to represent data flow, or to represent the fact that a port of a composite component is exported from one of its constituent components. For the Petri net procedures, there is no distinctionin both cases, the connected ports represent the same shared variable and are therefore modelled by the same, coalesced data place.

We can see this in figure 9. Connected outer ports of all the components are each represented by a single, shared data place. This holds not only for the dataflow of velocity and actualPosition from FQD to ProcessApplication but also for the setPoint port which is visible from the outside. Inner and outer ports of active and event components, on the other hand, are not coalesced, since they must be explicitly synchronized.

The figure also illustrates how composite components schedule their parts. The schedule, which is triggered by the start place of the composite, first fires FQD, then ProcessApplication, and finally ModBus. Since FQD and ModBus have independent behaviour (i.e., triggered by an event or running in a separate thread), the schedule is responsible only for synchronizing the data ports.

The constructed net is clearly deadlock-free: the only conflicts between simultaneously enabled transitions occur where sync transitions compete with critical sections of active components. Since each of these transitions lock all the required data ports simultaneously, no waits-for cycles are possible, and hence no deadlock can arise.

\subsection{Timing}

To construct a schedule for a composition of components, certain scheduling information must be associated with each subcomponent; this includes the worst-case execution time of the subcomponent and the desired cycle time. For an active subcomponent, this information must be provided separately for the sync and exec methods. It is also be necessary to specify a (partial) ordering for the execution of the subcomponents in a composition. 


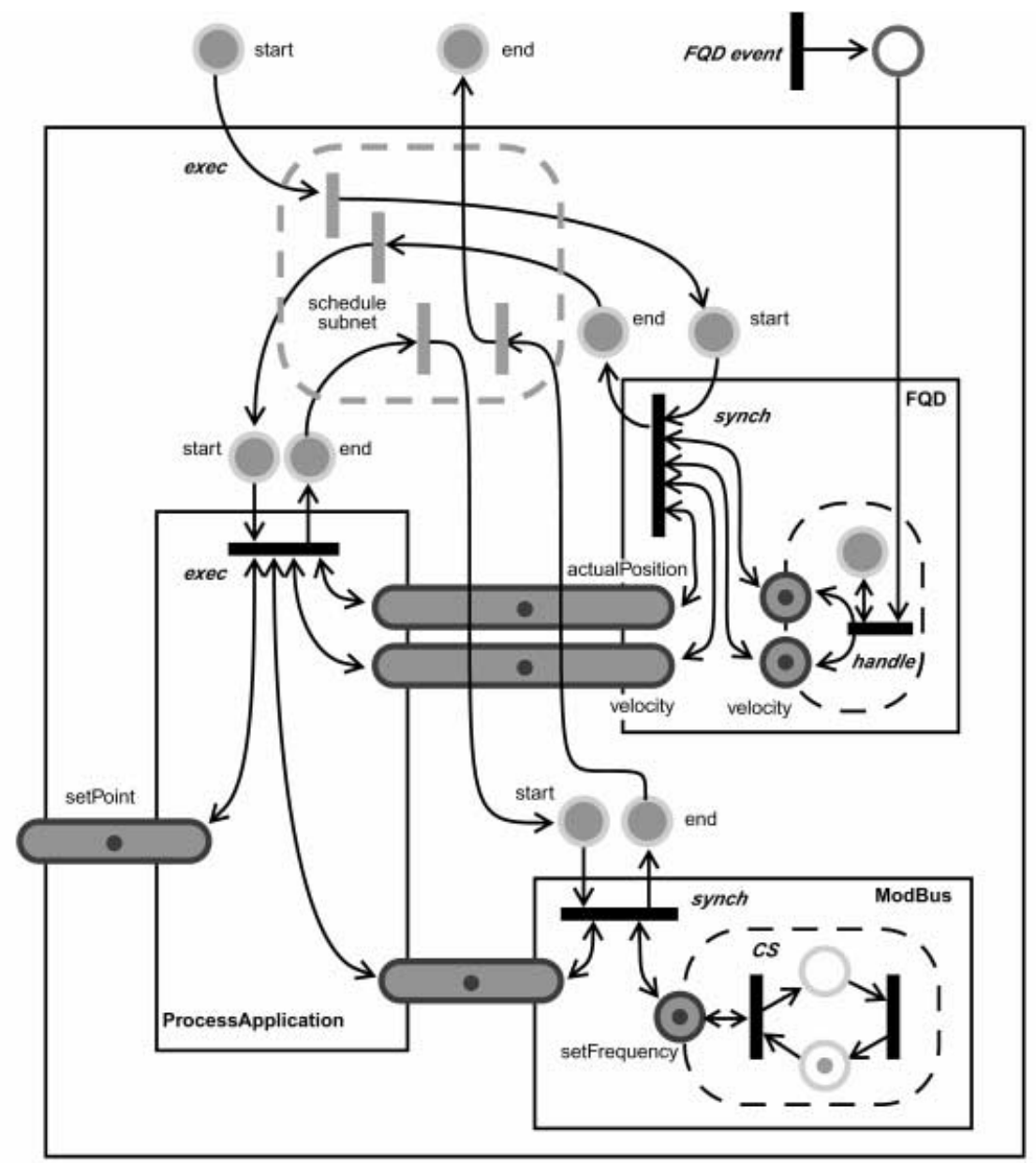

Figure 9 Petri net model of control loop example

The simplest form of schedule is a cyclic executive, in which components are wired together directly, such that each passes control to the next. Such schedules are very efficient, but are feasible only for the very simplest of compositions. Such a schedule can be represented in the Petri net model by inserting transitions between the end place of one component and the start place of the next.

So long as the schedule can be computed entirely statically, there is no need to explicitly represent the scheduling information for the components: it is represented implicitly in the schedule itself, but that schedule can be calculated off-line. However, it may still be desirable to verify the scheduler produced in this way. We have been investigating the use of time Petri nets [13] to represent the schedulers, and various Petri net tools such as Poses $++[3]$ and TPTPN [10] to assist in their analysis. We have also been investigating the use of hierarchical constraint solvers (such as Cassowary [1]), to cap- 
ture the timing requirements and partial ordering of components and check the feasibility of schedules, or even to generate them when this is possible.

\section{Status and Future Work}

In this paper we have presented some intermediate results of the PECOS project. The component model developed for PECOS addresses the requirements identified for the case study outlined above. Ongoing activities include: (i) formalization of the component model, (ii) investigation of various techniques, such as time Petri nets and hierarchical constraint solvers, to generate real-time schedules, (iii) implementation of components to support the PECOS case study demonstrator, (iv) implementation of the language mapping to generate executable code from specifications of component compositions.

\section{References}

[1] Greg J. Badros and Alan Borning, "The Cassowary Linear Arithmetic Constraint Solving Algorithm: Interface and Implementation,” Technical Report, no. UW Technical Report 98-06-04, University of Washington, 1998.

[2] Embedded C++ home page, www.caravan.net/ec2plus

[3] Gesellschaft für Prozeßautomation \& Consulting bH home page, www.gpc.de.

[4] PROFIBUS International, PA General Requirements, Version 3.0 (www.profibus.org)

[5] PROFIBUS International, Device Data Sheet for Actuators, Version 3.0

[6] Bastiaan Schönhage, "Model mapping to C++ or Java-based ultra-light environment", Pecos Deliverable D2.2.9-1, www.pecos-project.org

[7] Benedikt Schulz, Thomas Genssler, Alexander Christoph, Michael Winter, "Requirements for the Composition Environment", Pecos Deliverable D3.1, www.pecos-project.org

[8] Semiconductor Motorola Programming Note, Fast Quadrature Decode TPU Function (FQD), TPUPN02/D.

[9] Mary Shaw and David Garlan, Software Architecture: Perspectives on an Emerging Discipline, Prentice-Hall, 1996.

[10] TPTPN home page, www.diit.unict.it/users/scava/tptpn.html.

[11] Rob van Ommering, Jeff Kramer, Jeff Magee, "The Koala Component Model for Consumer Electronics Software”, IEEE Computer, March 2000, Vol. 33, No. 3, pp. 78-85.

[12] Jiacun Wang, Timed Petri Nets, Kluwer Academic Publishers, 1998.

[13] B. Berthomieu and M. Diaz. Modeling and verification of time dependent systems using time Petri nets. IEEE Transactions on Software Engineering, 17(3), pp. 259-273, 1991. 